\title{
Palladium-Catalyzed Carbene Insertion into Vinyl Halides and Trapping with Amines
}

\author{
Sean K. J. Devine and David L. Van Vranken*
}

\section{General Conditions}

\section{Supporting Information}

All reactions, unless specified, were carried out under an atmosphere of argon in glassware that was flame-dried under vacuum. All solvents, except those used in chromatography, were dried by passage over alumina as described by Grubbs. ${ }^{1}$ Amines were distilled from calcium hydride prior to use. Analytical thin layer chromatography was performed on EM Reagents $0.25 \mathrm{~mm}$ silica gel $60-\mathrm{F}$ plates. Liquid chromatography was performed using flash chromatography on SiliCycle silica gel $(40-63 \mu \mathrm{m})$ with the indicated solvent system. Trimethylsilyldiazomethane was used as purchased from Sigma. Commercial trimethylsilyldiazomethane varies in purity and often contains (chloromethyl)trimethylsilane. ${ }^{2}$

\section{Synthesis of Vinyl Halides}

1-(4-Iodobut-3-ynyl)benzene). ${ }^{3}$ A solution of 4-phenyl-1-butyne $(7.14 \mathrm{~g}, 54.8 \mathrm{mmol})$ in $25 \mathrm{~mL}$ of hexane was cooled to $-78^{\circ} \mathrm{C}$ and a solution of $2.3 \mathrm{M} n$-BuLi in hexane $(23.8 \mathrm{~mL}, 54.8 \mathrm{mmol})$ was then added dropwise over $30 \mathrm{~min}$. The resulting thick suspension was stirred at $-78{ }^{\circ} \mathrm{C}$ for 2 h. A solution of iodine $(13.9 \mathrm{~g}, 54.7 \mathrm{mmol})$ in $70 \mathrm{~mL}$ of dry ether was added over $45 \mathrm{~min}$. The cooling bath was removed and the reaction was allowed to warm to room temperature. Water $(35 \mathrm{~mL})$ was added and the resulting mixture was stirred for $10 \mathrm{~min}$. The aqueous layer was separated and extracted with $35 \mathrm{~mL}$ of hexane. The combined organic phases were washed with two $50 \mathrm{~mL}$ portions of water, $20 \mathrm{~mL}$ of $20 \%$ aqueous sodium thiosulfate, and then dried over anhydrous $\mathrm{MgSO}_{4}$. Solvent was removed by rotary evaporation and the residual oil was purified by silica gel chromatography using pentane to yield $11.9 \mathrm{~g}$ of 1-(4-iodobut-3-ynyl)benzene as a colorless oil (85\%). $\quad \mathrm{R}_{f}=0.42$ (hexanes); ${ }^{1} \mathrm{H}$ NMR $\left(500 \mathrm{MHz}, \mathrm{CDCl}_{3}\right) \delta$ 7.26-7.33 (m, 2H), $7.17-7.25(\mathrm{~m}, 3 \mathrm{H}), 2.83(\mathrm{t}, J=7.6 \mathrm{~Hz}, 2 \mathrm{H}), 2.63(\mathrm{t}, J=7.4 \mathrm{~Hz}, 2 \mathrm{H}) ;{ }^{13} \mathrm{C}$ NMR $(125 \mathrm{MHz})$ $\delta 140.4,128.7,128.6,126.6,94.1,35.1,23.3,-5.9$; IR (thin film) 3051, 2855, $2129 \mathrm{~cm}^{-1}$; LRMS (EI) 256(10), 129(38), 91(100).

1-((Z)-4-Iodobut-3-enyl)benzene) (1a). A solution of borane-dimethylsulfide complex (1.06 $\mathrm{mL}, 11.2 \mathrm{mmol})$ in $100 \mathrm{~mL}$ of ether was added to a flame-dried, two-necked, round-bottomed flask equipped with a thermometer, stirbar, and argon inlet. The solution was cooled to $0{ }^{\circ} \mathrm{C}$ and then cyclohexene $(2.27 \mathrm{~mL}, 22.4 \mathrm{mmol})$ was added over $10 \mathrm{~min}$. A white precipitate formed during the addition. The reaction mixture was allowed to warm to room temperature and stirred for $1 \mathrm{~h}$. The suspension was cooled to $0{ }^{\circ} \mathrm{C}$ and 1-(4-iodobut-3-ynyl)benzene $(2.87 \mathrm{~g}, 11.2$ mmol) was added slowly by syringe. The solution was allowed to warm to room temperature and was stirred for $1 \mathrm{~h}$. The solution was then cooled to $0{ }^{\circ} \mathrm{C}$ and $5.1 \mathrm{~mL}$ of AcOH was slowly added. The mixture was allowed to warm to room temperature and was stirred for $2 \mathrm{~h}$. Ether (10 $\mathrm{mL}$ ) was added and the solution was washed with four $7.5 \mathrm{~mL}$ portions of water, dried over anhydrous $\mathrm{MgSO}_{4}$, and concentrated by rotary evaporation to give a yellow oil, which was subsequently purified by silica gel chromatography using pentane to yield $1.82 \mathrm{~g}(63 \%)$ of $\mathbf{1 a}$ as a colorless oil. $\mathrm{R}_{f}=0.52$ (Hexanes); ${ }^{1} \mathrm{H}$ NMR $\left(500 \mathrm{MHz}, \mathrm{CDCl}_{3}\right) \delta 7.27-7.34(\mathrm{~m}, 2 \mathrm{H}), 7.18-$ 
$7.24(\mathrm{~m}, 3 \mathrm{H}), 6.18-6.25(\mathrm{~m}, 2 \mathrm{H}), 2.75(\mathrm{t}, J=8.5 \mathrm{~Hz}, 2 \mathrm{H}), 2.47(\mathrm{td}, J=6.5,1.5 \mathrm{~Hz}, 2 \mathrm{H}) ;{ }^{13} \mathrm{C}$ NMR (125 MHz) $\delta 141.1,140.3,128.5,128.5,126.2,83.2,36.7,34.4$; IR (thin film) 3062, 2855, 1943, $1604 \mathrm{~cm}^{-1}$; LRMS (EI) 258(5), 131(100), 91(98).

1-((E)-4-Iodo-3-methylbut-3-enyl)benzene $\quad(\mathbf{1 b}) .^{4} \quad$ To a slurry of dichlorobis $\left[\eta^{5}\right.$ cyclopentadienyl]zirconium $(11.0 \mathrm{~g}, 37.6 \mathrm{mmol})$ in dichloromethane $(90 \mathrm{~mL})$ was added trimethylalane $(7.21 \mathrm{~mL}, 75.2 \mathrm{mmol})$ at room temperature. To this solution was added 4-phenyl1-butyne (4.92 g, $37.8 \mathrm{mmol})$. After stirring the solution for $24 \mathrm{~h}$, iodine $(9.60 \mathrm{~g}, 37.8 \mathrm{mmol})$ dissolved in $50 \mathrm{~mL}$ of THF was added dropwise at $0{ }^{\circ} \mathrm{C}$. After the iodide color disappears the reaction was quenched with $20 \mathrm{~mL}$ of water and extracted with ether $(2 \mathrm{x} 40 \mathrm{~mL})$. The organic layer was dried over magnesium sulfate and concentrated by rotary evaporation. The residue was purified by silica chromatography using pentane to yield $7.71 \mathrm{~g}(75 \%)$ of $\mathbf{1 b}$ as a colorless oil. $\mathrm{R}_{f}=0.45$ (hexanes); ${ }^{1} \mathrm{H}$ NMR $\left(500 \mathrm{MHz}, \mathrm{CDCl}_{3}\right) \delta 7.26-7.30(\mathrm{~m}, 2 \mathrm{H}), 7.13-7.22(\mathrm{~m}, 3 \mathrm{H})$, $5.89(\mathrm{q}, J=1.2 \mathrm{~Hz} 1 \mathrm{H}), 2.72(\mathrm{t}, J=7.7 \mathrm{~Hz}, 2 \mathrm{H}), 2.49(\mathrm{t}, J=7.6 \mathrm{~Hz}, 2 \mathrm{H}), 1.9(\mathrm{~d}, 1.0 \mathrm{~Hz}, 3 \mathrm{H}) ;{ }^{13} \mathrm{C}$ NMR $(125 \mathrm{MHz}) \delta 147.4,141.3,128.6,128.4,126.2,75.6,41.6,34.5,24.2$; IR (thin film) 3035, 1943, $1604 \mathrm{~cm}^{-1}$; LRMS (EI) 272(33), 180(39), 144(100), 79(117), 90(91).

1-(3-Iodobut-3-enyl)benzene (1c). ${ }^{5}$ To a solution of $B$-iodo-9-BBN $(2.07 \mathrm{~mL}, 13.1 \mathrm{mmol})$ in pentane $(73 \mathrm{~mL})$ was slowly added 4-phenyl-1-butyne $(1.42 \mathrm{~g}, 10.9 \mathrm{mmol})$ at $-20{ }^{\circ} \mathrm{C}$. After stirring for $2 \mathrm{~h}$ at $-20^{\circ} \mathrm{C}$, acetic acid $(7 \mathrm{~mL})$ was added and the mixture was stirred for $1 \mathrm{~h}$ at 0 ${ }^{\circ} \mathrm{C}$. The mixture was warmed to room temperature and $30 \mathrm{~mL}$ of $2 \mathrm{M} \mathrm{NaOH}$ solution was added followed by $6 \mathrm{~mL}$ of $30 \%$ hydrogen peroxide. The mixture was stirred for $30 \mathrm{~min}$. The product was extracted with pentane, the combined organic layers washed with water, aqueous $\mathrm{NaHCO}_{3}$, and then dried over magnesium sulfate. The residue was concentrated by rotary evaporation and was purified by silica chromatography using pentane to yield $2.42 \mathrm{~g}(86 \%)$ of $\mathbf{1 c}$ as a colorless oil. $\mathrm{R}_{f}=0.49$ (pentane); ${ }^{1} \mathrm{H}$ NMR $\left(500 \mathrm{MHz} \mathrm{CDCl}_{3}\right) \delta 7.28-7.33(\mathrm{~m}, 2 \mathrm{H}), 7.20-7.25(\mathrm{~m}, 3 \mathrm{H})$, $5.97(\mathrm{~d}, J=1.5 \mathrm{~Hz}, 1 \mathrm{H}), 5.71(\mathrm{~d}, J=1.5 \mathrm{~Hz}, 1 \mathrm{H}), 2.86(\mathrm{t}, J=8.0 \mathrm{~Hz}, 2 \mathrm{H}), 2.71(\mathrm{t}, J=8.5 \mathrm{~Hz}$, $2 \mathrm{H}) ;{ }^{13} \mathrm{C}$ NMR (125 MHz) $\delta 140.3,128.7,128.5,126.3,126.2,111.1,47.3,35.6$; IR (thin film) 3026, 2926, 1801, $1617 \mathrm{~cm}^{-1}$; LRMS (EI) 258(79), 131(81), 91(100).

1-((Z)-4-Bromobut-3-enyl)benzene (1d). ${ }^{3}$ A solution of 4-phenyl-1-butyne (2.59 g, $\left.20.0 \mathrm{mmol}\right)$ in $10 \mathrm{~mL}$ of hexane was cooled to $-78{ }^{\circ} \mathrm{C}$ and a solution of $2.3 \mathrm{M} n$-BuLi in hexane $(8.70 \mathrm{~mL}$, $20.0 \mathrm{mmol}$ ) was then added dropwise over $30 \mathrm{~min}$. The resulting thick suspension was stirred at $-78{ }^{\circ} \mathrm{C}$ for $2 \mathrm{~h}$. Bromine $(1.03 \mathrm{~mL}, 20.0 \mathrm{mmol})$ was added over $45 \mathrm{~min}$. The cooling bath was removed and the reaction was allowed to warm to room temperature. Water $(35 \mathrm{~mL})$ was added and the resulting mixture was stirred for $10 \mathrm{~min}$. The aqueous layer was separated and extracted with $35 \mathrm{~mL}$ of hexane. The combined organic phases were washed with two $50 \mathrm{~mL}$ portions of water, $20 \mathrm{~mL}$ of $20 \%$ aqueous sodium thiosulfate, and then dried over anhydrous $\mathrm{MgSO}_{4}$. Solvent was removed by rotary evaporation and the residual oil was used without futher purification.

A solution of borane-dimethylsulfide complex $(2.07 \mathrm{~mL}, 22.0 \mathrm{mmol})$ in $180 \mathrm{~mL}$ of ether was added to a flame-dried, two-necked, round-bottomed flask equipped with a thermometer, stirbar, and argon inlet. The solution was cooled to $0{ }^{\circ} \mathrm{C}$ and then cyclohexene $(4.45 \mathrm{~mL}, 44.0 \mathrm{mmol})$ was added over $10 \mathrm{~min}$. A white precipitate formed during the addition. The reaction mixture was allowed to warm to room temperature and stirred for $1 \mathrm{~h}$. The suspension was cooled $0{ }^{\circ} \mathrm{C}$ and the crude alkynyl bromide was then added by syringe. The solution was allowed to warm to 
room temperature and was stirred for $1 \mathrm{~h}$. The solution was then cooled to $0{ }^{\circ} \mathrm{C}$ and $10.2 \mathrm{~mL}$ of $\mathrm{AcOH}$ was slowly added. The mixture was allowed to warm to room temperature and was stirred for $2 \mathrm{~h}$. Ether $(10 \mathrm{~mL})$ was added and the solution was washed with four $7.5 \mathrm{~mL}$ portions of water, dried over anhydrous $\mathrm{MgSO}_{4}$, and concentrated by rotary evaporation to give a yellow oil, which was subsequently purified by silica gel chromatography using pentane to give $\mathbf{1 d}$ as a cololess oil $(1.50 \mathrm{~g}, 36 \%)$. Spectral data was identical to that reported in the literature. ${ }^{6}{ }^{1} \mathrm{H} \mathrm{NMR}$ $\left(500 \mathrm{MHz}, \mathrm{CDCl}_{3}\right) \delta$ 7.27-7.31 (m, 2H), 7.19-7.23 (m, 3H), $6.17(\mathrm{dt}, J=7.0,1.1 \mathrm{~Hz}, 1 \mathrm{H}), 6.12$ $(\mathrm{q}, J=6.9 \mathrm{~Hz}, 1 \mathrm{H}), 2.74(\mathrm{t}, J=7.5 \mathrm{~Hz}, 2 \mathrm{H}), 2.53(\mathrm{tdd}, J=7.3,6.9,0.9 \mathrm{~Hz}, 2 \mathrm{H})$.

\section{Synthesis of Allylamines}

General Procedure for Three Component Coupling of Vinyl Halides, Amines, and Diazo Compounds. To a round bottom flask containing a magnetic stir bar was added vinyl halide, $\mathrm{Pd}_{2}(\mathrm{dba})_{3} \cdot \mathrm{CHCl}_{3}(2.5 \mathrm{~mol} \%)$, triphenylphosphine $(15 \mathrm{~mol} \%)$, and tetrahydofuran (to give $0.25 \mathrm{M}$ substrate). The flask was purged with argon and the purple suspension was stirred at room temperature until a clear orange solution was obtained (about $10 \mathrm{~min}$ ). Amine (400 mol\%) was then added by syringe and the green solution was heated to $46{ }^{\circ} \mathrm{C}$. Trimethylsilyldiazomethane (150 mol\%, $2 \mathrm{M}$ in hexanes) was added by syringe over $10 \mathrm{~h}$. The resulting orange solution was cooled to room temperature and poured into a separatory funnel containing aqueous $1 \%$ sodium hydroxide solution. The resulting mixture was extracted three times with ethyl acetate. The combined extracts were washed with brine, dried over magnesium sulfate and concentrated by rotary evaporation to give a yellow oil. The residue was purified by flash chromatography using the designated solvent system to afford the amine.

(E)- $N$-Benzyl-1-(trimethylsilyl)-5-phenylpent-1-en-3-amine (2a). To a $10 \mathrm{~mL}$ round bottom flak containing a magnetic stir bar was added vinyl iodide 1a $(0.190 \mathrm{~g}, 0.736 \mathrm{mmol})$, $\mathrm{Pd}_{2}(\mathrm{dba})_{3} \bullet \mathrm{CHCl}_{3}(0.019 \mathrm{~g}, 0.018 \mathrm{mmol})$, triphenylphosphine $(0.029 \mathrm{~g}, 0.11 \mathrm{mmol})$, and charged with tetrahydofuran $(2.9 \mathrm{~mL})$. The flask was purged with argon and the purple suspension was stirred at room temperature until a clear orange solution was obtained (10 min). Benzylamine $(0.32 \mathrm{~mL}, 2.94 \mathrm{mmol})$ was then added by syringe and the green solution was heated to $46{ }^{\circ} \mathrm{C}$. Trimethylsilyldiazomethane $(0.55 \mathrm{~mL}, 2 \mathrm{M}$ in hexanes) was added by syringe over $10 \mathrm{~h}$. The resulting orange solution was cooled to room temperature, and poured into a separatory funnel containing $3 \mathrm{~mL}$ of aqueous $1 \%$ sodium hydroxide solution. The resulting mixture was extracted three times with ethyl acetate $(5 \mathrm{~mL})$. The combined extracts were washed with brine $(1 \mathrm{~mL})$, dried over magnesium sulfate and concentrated by rotary evaporation to give a yellow oil. The residue was purified by flash chromatography using 15:85:1 ethyl acetate/hexanes/ $\mathrm{Et}_{3} \mathrm{~N}$ to yield amine $2 \mathbf{a}$ as a colorless oil $(0.045 \mathrm{~g}, 19 \%) . \mathrm{R}_{f}=0.15-35$ (15:85 ethyl acetate/hexanes); ${ }^{1} \mathrm{H}$ NMR $\left(500 \mathrm{MHz}, \mathrm{CDCl}_{3}\right) \delta$ 7.17-7.30 (m, 7H), 7.08-7.15 (m, 3H), 5.81 (dd, J=18.6, 7.5 Hz, 1H), 5.72 $(\mathrm{d}, J=18.6 \mathrm{~Hz}, 1 \mathrm{H}), 3.75(\mathrm{~d}, J=13.1 \mathrm{~Hz}, 1 \mathrm{H}), 3.59(\mathrm{~d}, J=13.1 \mathrm{~Hz}, 1 \mathrm{H}), 3.03(\mathrm{q}, J=6.9 \mathrm{~Hz}$, $1 \mathrm{H}), 2.60$ (ddd, $J=12.0,6.5,2.6 \mathrm{~Hz}, 2 \mathrm{H}), 1.67-1.82(\mathrm{~m}, 2 \mathrm{H}), 1.15-1.35$ (br, $1 \mathrm{H}), 0.05$ (s, 9H); ${ }^{13} \mathrm{C}$ NMR $(125 \mathrm{MHz}) \delta 148.7,142.3,140.8,132.1,128.6,128.5,128.5,128.4,127.0,125.9,63.1$, 51.5, 37.2, 32.4, -0.9; IR (thin film) 3307, 3026, 2952, 1604, $1247 \mathrm{~cm}^{-1}$; HRMS (EI) $\mathrm{m} / z$ calcd for $\mathrm{C}_{21} \mathrm{H}_{29} \mathrm{NSi}[\mathrm{M}+\mathrm{H}]^{+} 324.2148$, found 324.2141.

1-((E)-1-(Trimethylsilyl)-5-phenylpentyl-1-en-3-yl)pyrrolidine (2b). Following the general procedure, pyrrolidine $(0.44 \mathrm{~mL}, 5.36 \mathrm{mmol})$ and vinyl iodide $1 \mathrm{a}(0.346 \mathrm{~g}, 1.34 \mathrm{mmol})$ gave amine $\mathbf{2 b}$ as a colorless oil $(0.103 \mathrm{~g}, 27 \%) . \quad \mathrm{R}_{f}=0.16-0.35\left(25: 75\right.$ ethyl acetate/hexanes); ${ }^{1} \mathrm{H}$ 
NMR (500 MHz, $\left.\mathrm{CDCl}_{3}\right) \delta 7.22-7.31(\mathrm{~m}, 2 \mathrm{H}), 7.13-7.20(\mathrm{~m}, 3 \mathrm{H}), 5.96(\mathrm{dd}, J=19.0,8.5 \mathrm{~Hz}$, $1 \mathrm{H}), 5.76(\mathrm{~d}, J=19.5 \mathrm{~Hz}, 1 \mathrm{H}), 2.62-2.65(\mathrm{~m}, 2 \mathrm{H}), 2.48-2.52(\mathrm{~m}, 5 \mathrm{H}), 2.01-2.08(\mathrm{~m}, 1 \mathrm{H}), 1.68-$ $1.83(\mathrm{~m}, 2 \mathrm{H}), 1.71-1.81(\mathrm{~m}, 4 \mathrm{H}), 1.59-1.68(\mathrm{~m}, 1 \mathrm{H}), 0.09(\mathrm{~s}, 9 \mathrm{H}) ;{ }^{13} \mathrm{C} \mathrm{NMR}(125 \mathrm{MHz})$ $\delta 143.2,139.6,137.4,128.7,128.4,126.5,70.1,51.8,50.7,31.9,31.7,23.4,23.3,-1.4$; IR (thin film) 3026, 2954, 1939, 1604, $1247 \mathrm{~cm}^{-1}$; HRMS (EI) $\mathrm{m} / z$ calcd for $\mathrm{C}_{18} \mathrm{H}_{29} \mathrm{NSi}[\mathrm{M}+\mathrm{H}]^{+}$ 288.2148, found 288.2139.

1-((E)-1-(Trimethylsilyl)-5-phenylpentyl-1-en-3)piperidine (2c). Following the general procedure, piperidine $(0.401 \mathrm{~g}, 4.06 \mathrm{mmol})$ and vinyl iodide $1 \mathbf{a}(0.262 \mathrm{~g}, 1.01 \mathrm{mmol})$ gave amine 2c as a colorless oil $(0.279 \mathrm{~g}, 91 \%) . \mathrm{R}_{f}=0.17-0.33$ (10:90 ethyl acetate/hexanes); ${ }^{1} \mathrm{H}$ NMR (500 $\left.\mathrm{MHz}, \mathrm{CDCl}_{3}\right) \delta 7.23-7.28(\mathrm{~m}, 2 \mathrm{H}), 7.13-7.19(\mathrm{~m}, 3 \mathrm{H}), 5.94(\mathrm{dd}, J=18.1,8.5 \mathrm{~Hz}, 1 \mathrm{H}), 5.71(\mathrm{~d}, J$ $=18.1 \mathrm{~Hz}, 1 \mathrm{H}), 2.75(\mathrm{dt}, J=8.6,5.1 \mathrm{~Hz}, 1 \mathrm{H}), 2.58-2.66(\mathrm{~m}, 1 \mathrm{H}), 2.46-2.56(\mathrm{~m}, 3 \mathrm{H}), 2.33-2.41$ $(\mathrm{m}, 2 \mathrm{H}), 1.98(\mathrm{ddt}, J=13.2,10.1,5.4 \mathrm{~Hz} 1 \mathrm{H}), 1.67-1.77(\mathrm{~m}, 1 \mathrm{H}), 1.49-1.63(\mathrm{~m}, 4 \mathrm{H}), 1.41(\mathrm{dd} J$

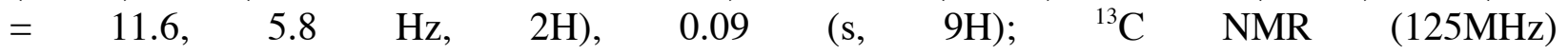
$\delta 145.7,142.8,133.7,128.6,128.4,125.7,70.8,50.9,33.6,32.9,26.6,25.0,-0.9$; IR (thin film) 3026, 2854, 1799, 1605, $1246 \mathrm{~cm}^{-1}$; HRMS (EI) $\mathrm{m} / z$ calcd for $\mathrm{C}_{19} \mathrm{H}_{31} \mathrm{NSi}[\mathrm{M}+\mathrm{H}]^{+} 302.2304$, found 302.2303 .

1-((E)-1-(Trimethylsilyl)-5-phenylpentyl-1-en-3)morpholine (2d). Following the general procedure, morpholine $(0.99 \mathrm{~mL}, 11.5 \mathrm{mmol})$ and vinyl iodide $1 \mathbf{a}(0.373 \mathrm{~g}, 1.44 \mathrm{mmol})$ gave amine $2 d$ as a colorless oil $(0.402 \mathrm{~g}, 92 \%) . \quad \mathrm{R}_{f}=0.18-0.34\left(15: 85\right.$ ethyl acetate/hexanes); ${ }^{1} \mathrm{H}$ NMR (500 MHz, $\left.\mathrm{CDCl}_{3}\right) \delta$ 7.26-7.32 (m, 2H), 7.16-7.22 (m, 3H), $5.92(\mathrm{dd}, J=18.7,8.2 \mathrm{~Hz}$, $1 \mathrm{H}), 5.78(\mathrm{~d}, J=18.7 \mathrm{~Hz}, 1 \mathrm{H}), 3.67-3.77(\mathrm{~m}, 4 \mathrm{H}), 2.76(\mathrm{ddd}, J=5.4,7.9,8.2 \mathrm{~Hz} 1 \mathrm{H}), 2.62-2.70$ $(\mathrm{m}, 1 \mathrm{H}), 2.52-2.62(\mathrm{~m}, 3 \mathrm{H}), 2.42-2.50(\mathrm{~m}, 2 \mathrm{H}), 1.97(\mathrm{ddt}, J=12.9,9.9,5.9 \mathrm{~Hz} \mathrm{1H}), 1.70-1.79$ $(\mathrm{m}, 1 \mathrm{H}), 0.11(\mathrm{~s}, 9 \mathrm{H}) ;{ }^{13} \mathrm{C}$ NMR $(125 \mathrm{MHz}) \delta 144.9,142.5,134.9,128.5,128.4,125.8,70.6$, 67.5, 50.4, 33.1, 32.5, -1.0; IR (thin film) 3026, 2954, 1865, 1604, $1248 \mathrm{~cm}^{-1}$; HRMS (EI) $\mathrm{m} / z$ calcd for $\mathrm{C}_{18} \mathrm{H}_{29} \mathrm{NOSi}[\mathrm{M}+\mathrm{H}]^{+}$304.2097, found 304.2098.

1-((E)-3-Methyl-1-(trimethylsilyl)-5-phenylpent-1-en-3-yl)piperidine (3). Following the general procedure, piperidine $(0.72 \mathrm{~mL}, 7.32 \mathrm{mmol})$ and vinyl iodide $\mathbf{1 b}(0.497 \mathrm{~g}, 1.83 \mathrm{mmol})$ gave amine 3 as a colorless oil $(0.304 \mathrm{~g}, 53 \%) . \mathrm{R}_{f}=0.20-0.37\left(10: 90\right.$ ethyl acetate/hexanes); ${ }^{1} \mathrm{H}$ NMR (500 MHz, $\left.\mathrm{CDCl}_{3}\right) \delta 7.26-7.30(\mathrm{~m}, 2 \mathrm{H}), 7.16-7.20(\mathrm{~m}, 3 \mathrm{H}), 6.07(\mathrm{~d}, J=19.4 \mathrm{~Hz}, 1 \mathrm{H})$, $5.70(\mathrm{~d}, J=19.4 \mathrm{~Hz}, 1 \mathrm{H}), 2.48-2.57(\mathrm{~m}, 6 \mathrm{H}), 1.76(\mathrm{tt}, J=9.7,6.0 \mathrm{~Hz}, 2 \mathrm{H}), 1.54-1.57(\mathrm{~m}, 4 \mathrm{H})$,

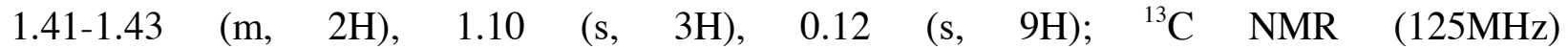
$\delta$ 153.01, 143.5, 128.4, 128.0, 125.6, 62.2, 47.3, 41.5, 30.6, 27.1, 25.2, 16.3, -0.9; IR (thin film) 3025, 2931, 1938, 1606, $1246 \mathrm{~cm}^{-1}$; HRMS (EI) $\mathrm{m} / z$ calcd for $\mathrm{C}_{20} \mathrm{H}_{33} \mathrm{NSi}[\mathrm{M}+\mathrm{H}]^{+} 316.2461$, found 316.2454 .

1-((E/Z)-2-(Trimethylsilyl)methylene-4-phenylbutyl)piperidine (4). Following the general procedure, piperidine $(0.393 \mathrm{~mL}, 3.98 \mathrm{mmol})$ and vinyl iodide $1 \mathrm{c}(0.257 \mathrm{~g}, 1.00 \mathrm{mmol})$ gave amine 4 as a 3.4:1 mixture of $E$ and $Z$ isomers $(0.054 \mathrm{~g}, 18 \%) . \mathrm{R}_{f}=0.16-0.31$ (10:90 ethyl acetate/hexanes); $(E){ }^{1} \mathrm{H}$ NMR $\left(500 \mathrm{MHz}, \mathrm{CDCl}_{3}\right) \delta$ 7.25-7.31 (m, 2H), 7.14-7.23 (m, 3H), 5.45 $(\mathrm{s}, 1 \mathrm{H}), 2.90(\mathrm{~s}, 2 \mathrm{H}), 2.65-2.77(\mathrm{~m}, 2 \mathrm{H}), 2.40-2.24(\mathrm{~m}, 2 \mathrm{H}), 2.32-2.36(\mathrm{~m}, 4 \mathrm{H}), 1.50-1.61(\mathrm{~m}$, 4H), 1.38-1.47 (m, 2H), $0.10(\mathrm{~s}, 9 \mathrm{H}) ;(Z){ }^{1} \mathrm{H}$ NMR $\left(500 \mathrm{MHz}, \mathrm{CDCl}_{3}\right) \delta$ 7.25-7.31 (m, 2H), 7.14-7.23 (m, 3H), 5.37 (s, 1H), $2.94(\mathrm{~s}, 2 \mathrm{H}), 2.65-2.77(\mathrm{~m}, 2 \mathrm{H}), 2.40-2.24(\mathrm{~m}, 2 \mathrm{H}), 2.32-2.36$ $(\mathrm{m}, 4 \mathrm{H}), \quad 1.50-1.61(\mathrm{~m}, 4 \mathrm{H}), 1.38-1.47(\mathrm{~m}, 2 \mathrm{H}), 0.10 \quad(\mathrm{~s}, 9 \mathrm{H}) ;{ }^{13} \mathrm{C}$ NMR $(125 \mathrm{MHz})$ 
$\delta$ 156.9, 155.8, 142.9, 142.7, 128.6, 128.4, 128.3, 126.4, 125.9, 125.9, 125.7, 67.5, 63.2, 54.9, 54. 7, 40.1, 36.7, 35.7, 34.9, 26.4, 24.7, 24.6, 0.9, 0.5; IR (thin film) 3026, 2934, 1614, 1453, 1247 $\mathrm{cm}^{-1}$; HRMS (EI) $\mathrm{m} / z$ calcd for $\mathrm{C}_{19} \mathrm{H}_{31} \mathrm{NSi}[\mathrm{M}+\mathrm{H}]^{+} 302.2304$, found 302.2301 .

1-((E)-1-(Trimethylsilyl)-5-phenylpentyl-1-en-3)piperidine (2c). Following the general procedure, piperidine $(0.288 \mathrm{~mL}, 2.92 \mathrm{mmol})$ and vinyl bromide $1 \mathbf{d}(0.154 \mathrm{~g}, 0.73 \mathrm{mmol})$ gave amine $2 \mathrm{c}$ as a colorless oil $(0.121 \mathrm{~g}, 55 \%) . \quad \mathrm{R}_{f}=0.17-0.33$ (10:90 ethyl acetate/hexanes); ${ }^{1} \mathrm{H}$ NMR (500 MHz, $\left.\mathrm{CDCl}_{3}\right) \delta$ 7.23-7.28 (m, 2H), 7.13-7.19 (m, 3H), $5.94(\mathrm{dd}, J=18.1,8.5 \mathrm{~Hz}$, $1 \mathrm{H}), 5.71(\mathrm{~d}, J=18.1 \mathrm{~Hz}, 1 \mathrm{H}), 2.75(\mathrm{dt}, J=8.6,5.1 \mathrm{~Hz}, 1 \mathrm{H}), 2.58-2.66(\mathrm{~m}, 1 \mathrm{H}), 2.46-2.56(\mathrm{~m}$, $3 \mathrm{H}), 2.33-2.41(\mathrm{~m}, 2 \mathrm{H}), 1.98$ (ddt, $J=13.2,10.1,5.4 \mathrm{~Hz} 1 \mathrm{H}), 1.67-1.77(\mathrm{~m}, 1 \mathrm{H}), 1.49-1.63(\mathrm{~m}$, $4 \mathrm{H}), \quad 1.41 \quad(\mathrm{dd} J=11.6, \quad 5.8 \mathrm{~Hz}, \quad 2 \mathrm{H}), \quad 0.09 \quad(\mathrm{~s}, \quad 9 \mathrm{H}) ;{ }^{13} \mathrm{C} \quad \mathrm{NMR} \quad(125 \mathrm{MHz})$ $\delta$ 145.7, 142.8, 133.7, 128.6, 128.4, 125.7, 70.8, 50.9, 33.6, 32.9, 26.6, 25.0, -0.9; IR (thin film) 3026, 2854, 1799, 1605, $1246 \mathrm{~cm}^{-1}$; HRMS (EI) $\mathrm{m} / z$ calcd for $\mathrm{C}_{19} \mathrm{H}_{31} \mathrm{NSi}[\mathrm{M}+\mathrm{H}]^{+} 302.2304$, found 302.2303 .

1-(3-methyl-5-phenylpent-1-en-3-yl)piperidine (5). To a $5 \mathrm{~mL}$ round bottom flask containg a magnetic stir bar was added allylamine $3(59 \mathrm{mg}, 0.19 \mathrm{mmol})$ and $0.5 \mathrm{~mL}$ of a $12 \mathrm{M} \mathrm{HCl}$ solution. The flask was equipped with a coldfinger and stirred at $100{ }^{\circ} \mathrm{C}$ for $14 \mathrm{~h}$. The reaction mixture was made basic by slowly adding $3.5 \mathrm{~mL}$ of a $2 \mathrm{M} \mathrm{NaOH}$ solution, extracted with ether $(3 \times 5 \mathrm{~mL})$, washed with water, dried over magnesium sulfate and concentrated by rotary evaporation to give a clear oil. The residue was purified by flash chromatography using 10:80:1 ethyl acetate/hexanes/Et $3 \mathrm{~N}$ to yield amine 5 as a colorless oil $(45 \mathrm{mg}, 96 \%) . \quad \mathrm{R}_{f}=0.10-0.23$ (10:90 ethyl acetate/hexanes) ${ }^{1} \mathrm{H}$ NMR $\left(500 \mathrm{MHz}, \mathrm{CDCl}_{3}\right) \delta$ 7.28-7.23 (m, 2H), 7.14-7.18 (m, $3 \mathrm{H}), 5.9(\mathrm{dd}, J=17.5,10.9 \mathrm{~Hz}, 1 \mathrm{H}), 5.14,(\mathrm{dd}, J=10.9,0.9 \mathrm{~Hz}, 1 \mathrm{H}), 5.05(\mathrm{dd}, 17.6,0.9 \mathrm{~Hz}, 1 \mathrm{H})$, 2.59-2.66 (m, 1H), 2.46-2.58 (m, 5H), 1.71-1.82 (m, 2H), 1.52-1.59 (m, 4H), 1.39-1.46 (m, 2H), 1.10 (s, 3H); ${ }^{13} \mathrm{C}$ NMR $(125 \mathrm{MHz}) \delta 155.9,145.1,143.6,128.4,125.6,113.4,47.2,41.7,30.5$, 27.1, 25.3, 16.7; IR (thin film) 3083, 2931, 1637, $1247 \mathrm{~cm}^{-1}$; HRMS (EI) $\mathrm{m} / z$ calcd for $\mathrm{C}_{17} \mathrm{H}_{25} \mathrm{~N}$ $[\mathrm{M}+\mathrm{H}]^{+}$244.2065, found 244.2061.

(E)-Ethyl 4-morpholino-6-phenylhex-2-enoate (6). Following the general procedure, vinyl iodide $1 \mathrm{a}(0.245 \mathrm{~g}, 0.949 \mathrm{mmol})$, morpholine $(0.60 \mathrm{~mL}, 7.59 \mathrm{mmol})$, ethyldiazoacetate $(0.15 \mathrm{~mL}$, $1.42 \mathrm{mmol}$ ) combined to give amine $\mathbf{6}$ as a colorless oil $(0.178 \mathrm{~g}, 62 \%) . \mathrm{R}_{f}=0.30$ (30:70 ethyl acetate/hexanes); ${ }^{1} \mathrm{H}$ NMR (500 MHz, $\mathrm{CDCl}_{3}$ ) $\delta 7.25-7.30(\mathrm{~m}, 2 \mathrm{H}), 7.14-7.21$ (m, 3H), 6.90 (dd, $J=15.8,8.8 \mathrm{~Hz}, 1 \mathrm{H}), 5.90(\mathrm{~d}, J=15.8 \mathrm{~Hz}, 1 \mathrm{H}), 4.21(\mathrm{q}, J=7.1 \mathrm{~Hz}, 2 \mathrm{H}), 3.66-3.74(\mathrm{~m}, 4 \mathrm{H})$, $3.03(\mathrm{q}, J=6.9 \mathrm{~Hz}, 1 \mathrm{H}), 2.95(\mathrm{dt}, J=8.7,5.7 \mathrm{~Hz}, 1 \mathrm{H}), 2.64-2.71(\mathrm{~m}, 1 \mathrm{H}), 2.54-2.62(\mathrm{~m}, 3 \mathrm{H})$, $2.45-2.51(\mathrm{~m}, 2 \mathrm{H}), 1.97-2.06(\mathrm{~m}, 1 \mathrm{H}), 1.74-1.82(\mathrm{~m}, 1 \mathrm{H}), 1.30(\mathrm{t}, J=7.0,3 \mathrm{H}) ;{ }^{13} \mathrm{C}$ NMR (125MHz) $\delta 166.0,147.2,141.7,128.5,128.5,126.1,124.1,67.4,65.6,60.9,50.0,32.7,32.2$, 14.4; IR (thin film) 2955, 1718, 1653, $1268 \mathrm{~cm}^{-1}$; HRMS (EI) $\mathrm{m} / z$ calcd for $\mathrm{C}_{18} \mathrm{H}_{25} \mathrm{NO}_{3}[\mathrm{M}+\mathrm{H}]^{+}$ 304.1913, found 304.1904. 


\section{References}

1. Pangborn, A. B.; Giardello, M. A.; Grubbs, R. H.; Rosen, R. K.; Timmers, F. J. Organometallics 1996, 15, 1518-1520.

2. Lebel, H.; Paquet, V. Organometallics 2004, 23, 1187-1190.

3. Denmark, S. E.; Wang, Z. Org. Synth. 2005, 81, 42-53.

4. Negishi, E.; Van Horn, D. E.; Yoshida, T. J. Am. Chem. Soc. 1985, 107, 6639-6647.

5. Hara, S.; Dojo, H.; Takinami, S.; Suzuki, A. Tetrahedron Lett. 1983, 24, 731-734.

6. Uenishi, J.; Kawahama, R.; Yonemitsu, O. J. Org. Chem. 1998, 63, 8965-8975. 

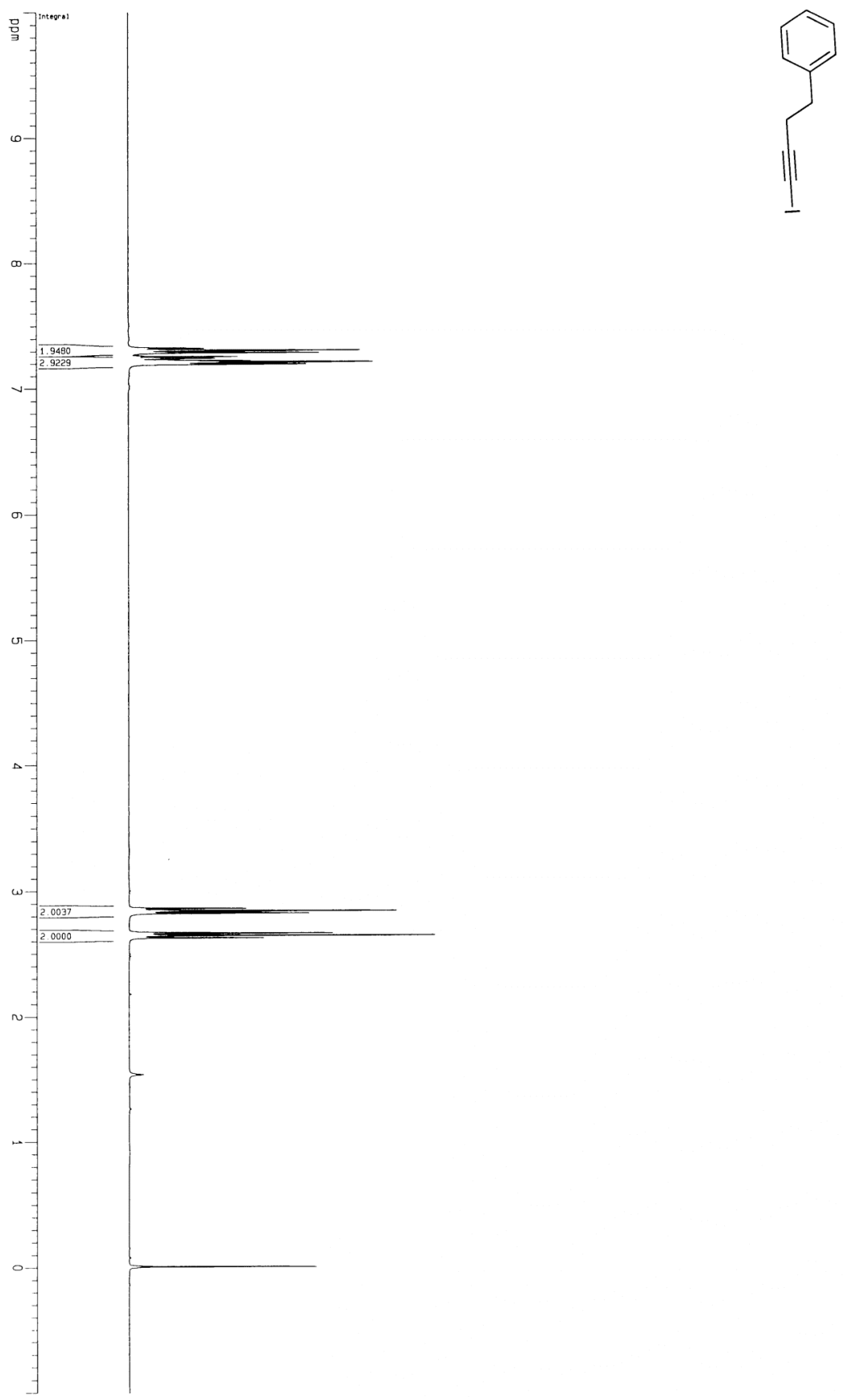


$$
F
$$




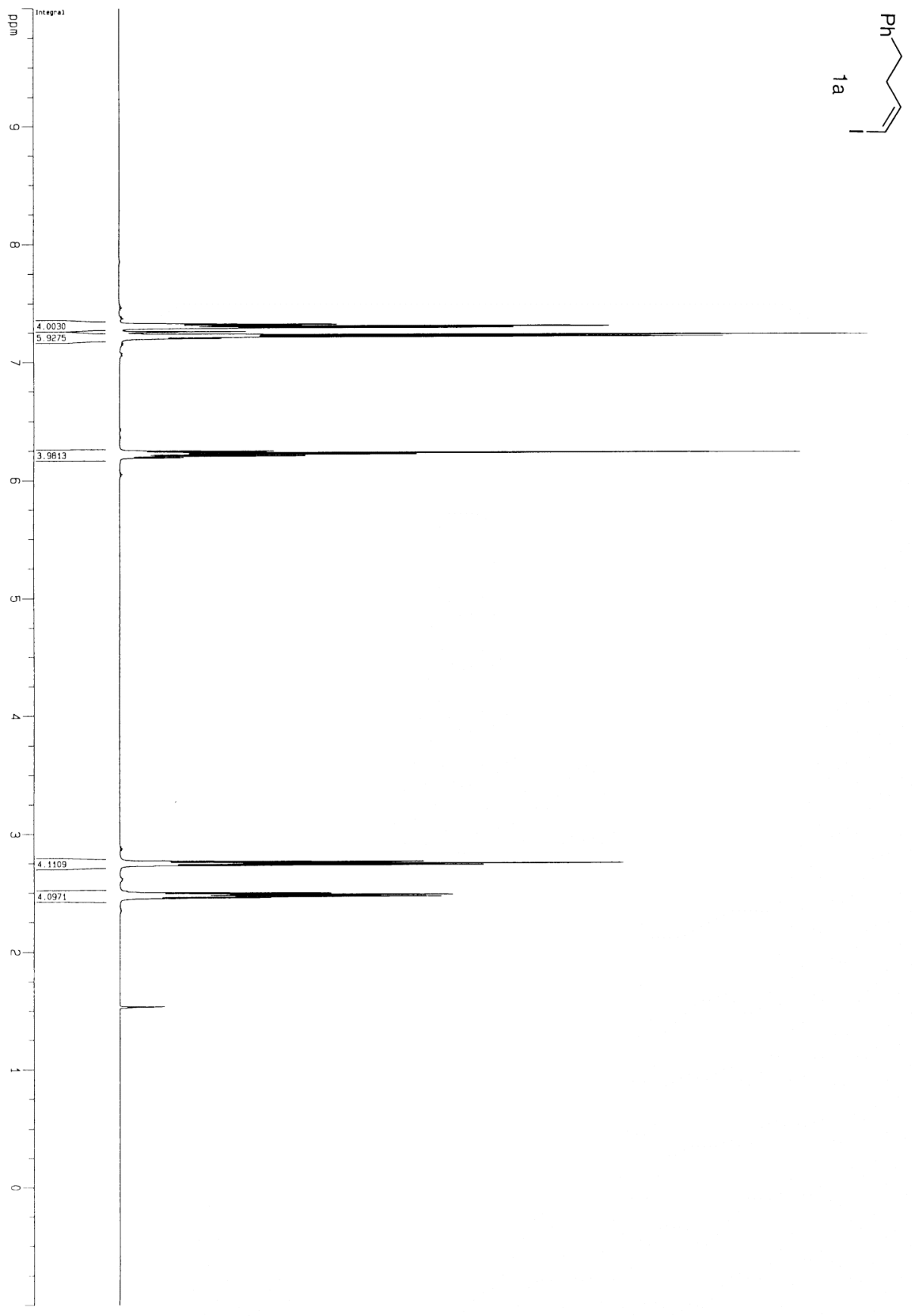




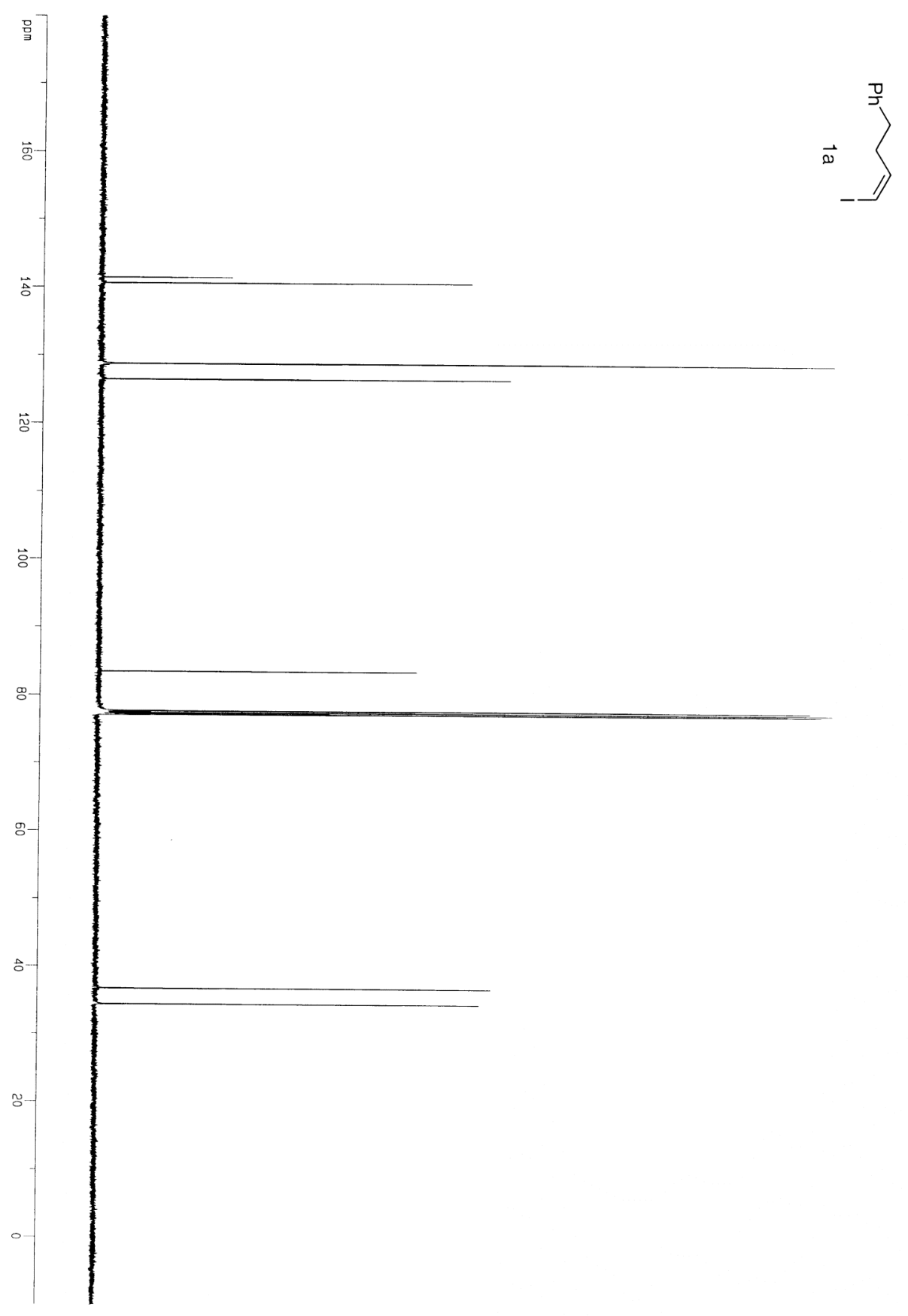




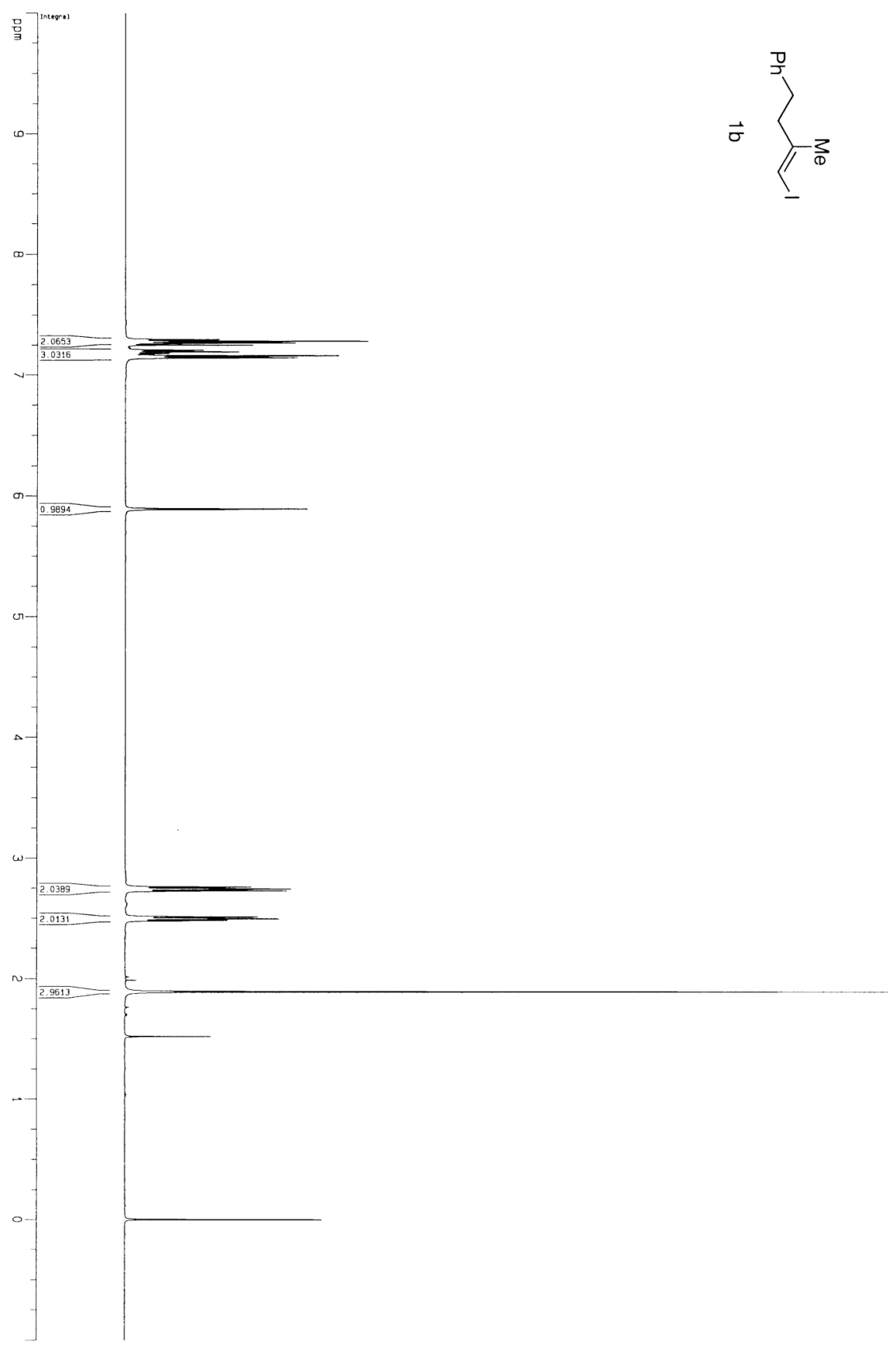




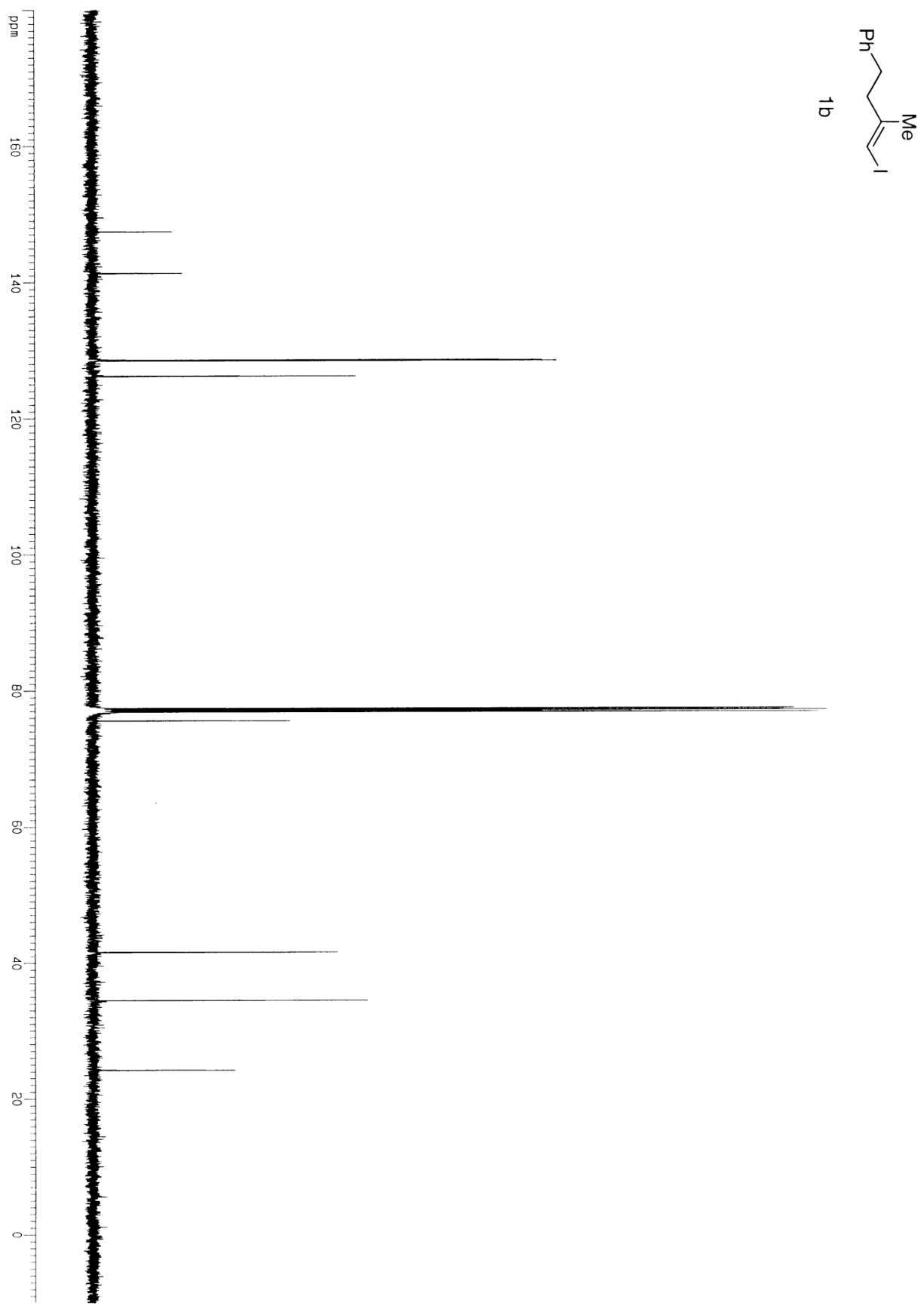

S12 


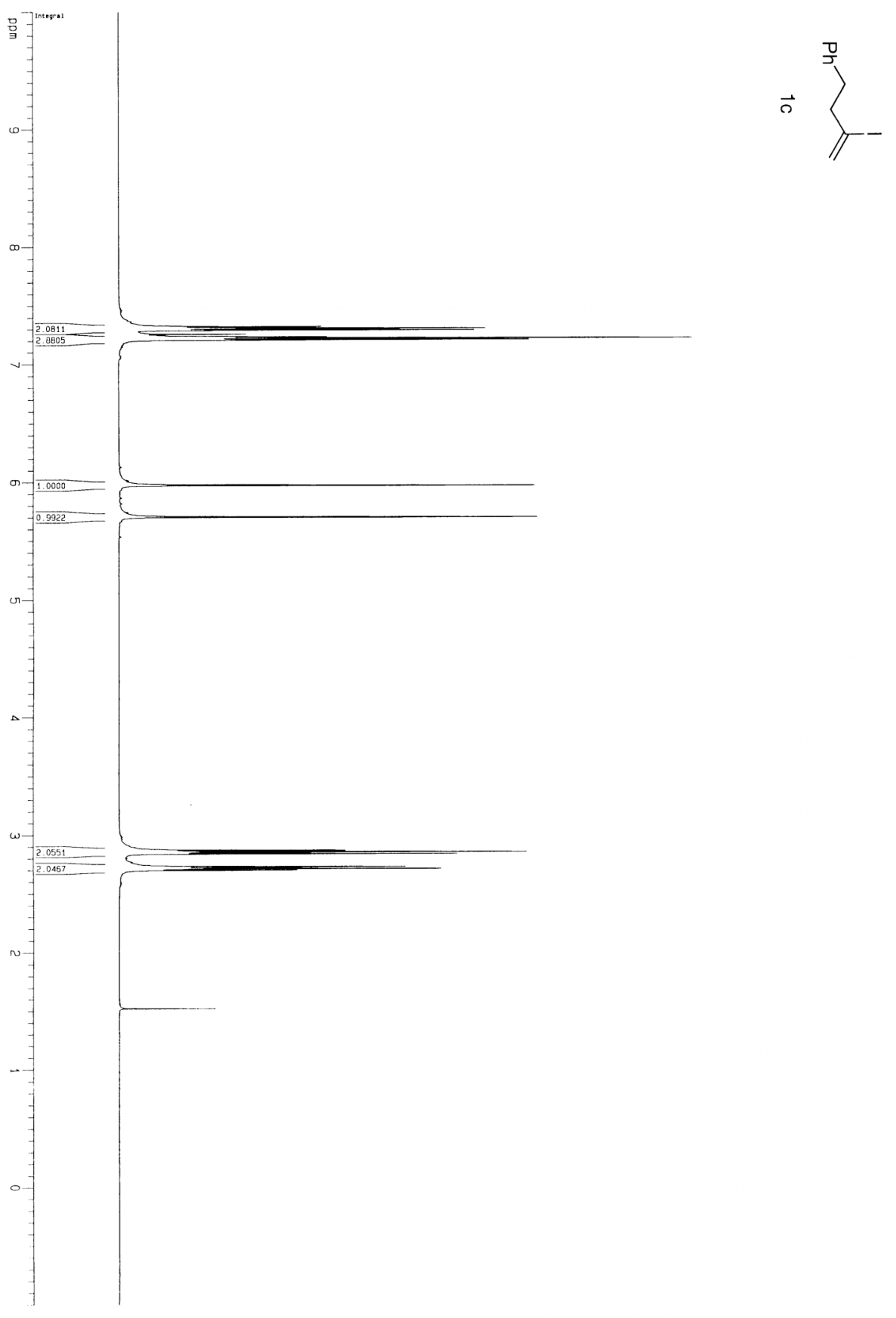




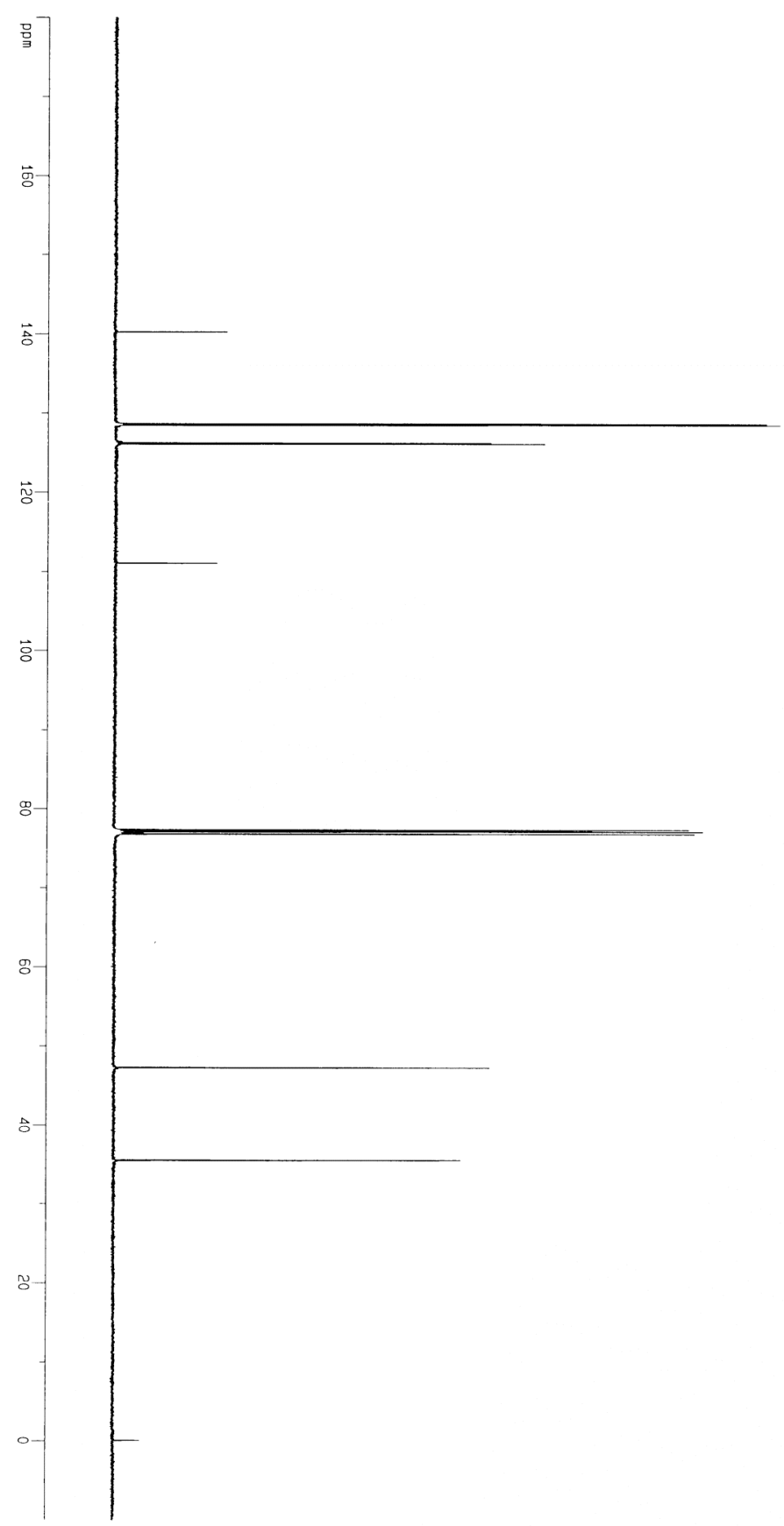

$\overrightarrow{0} \sum_{--}^{0}$ 


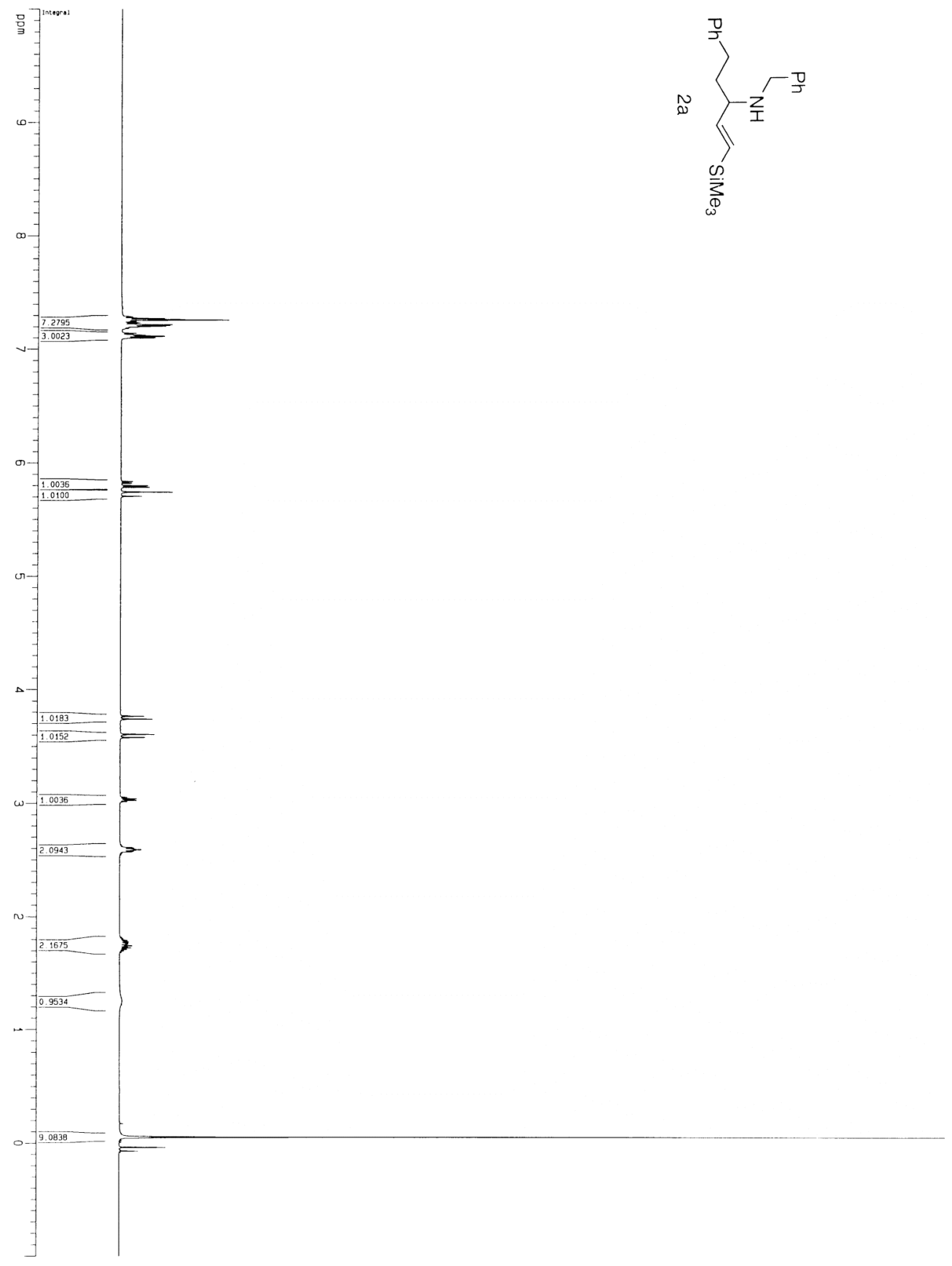




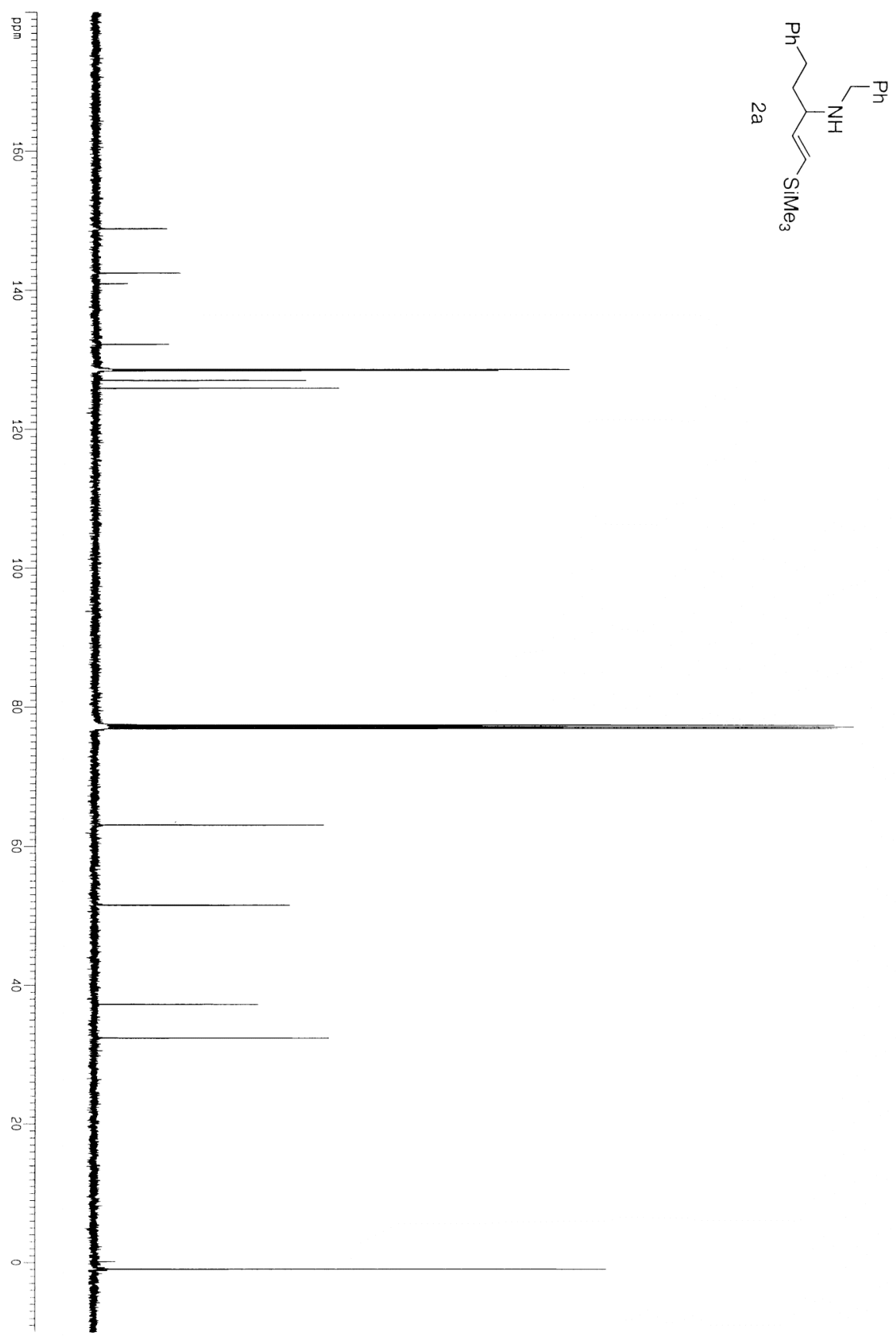

S16 


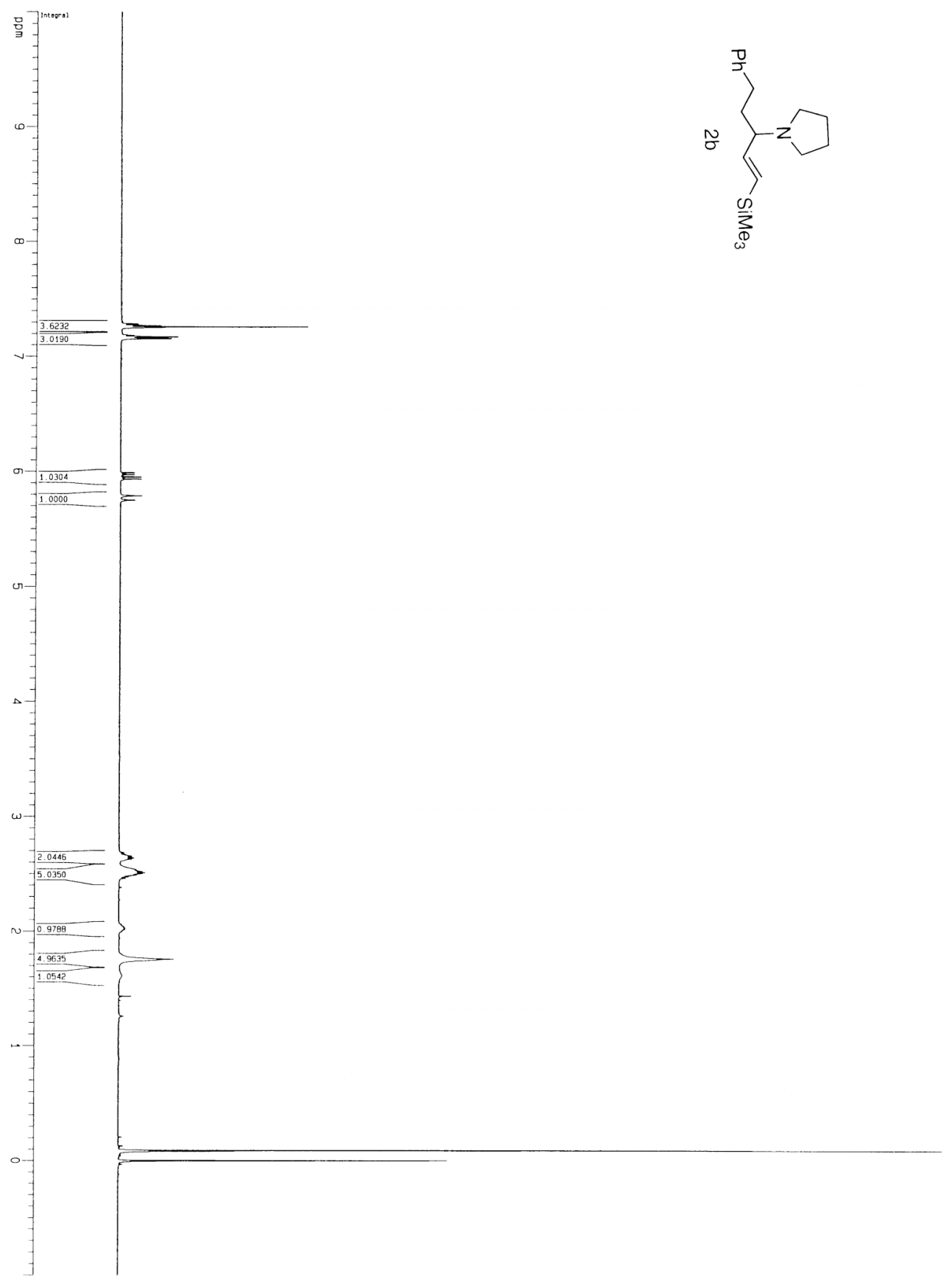




$$
\mid
$$




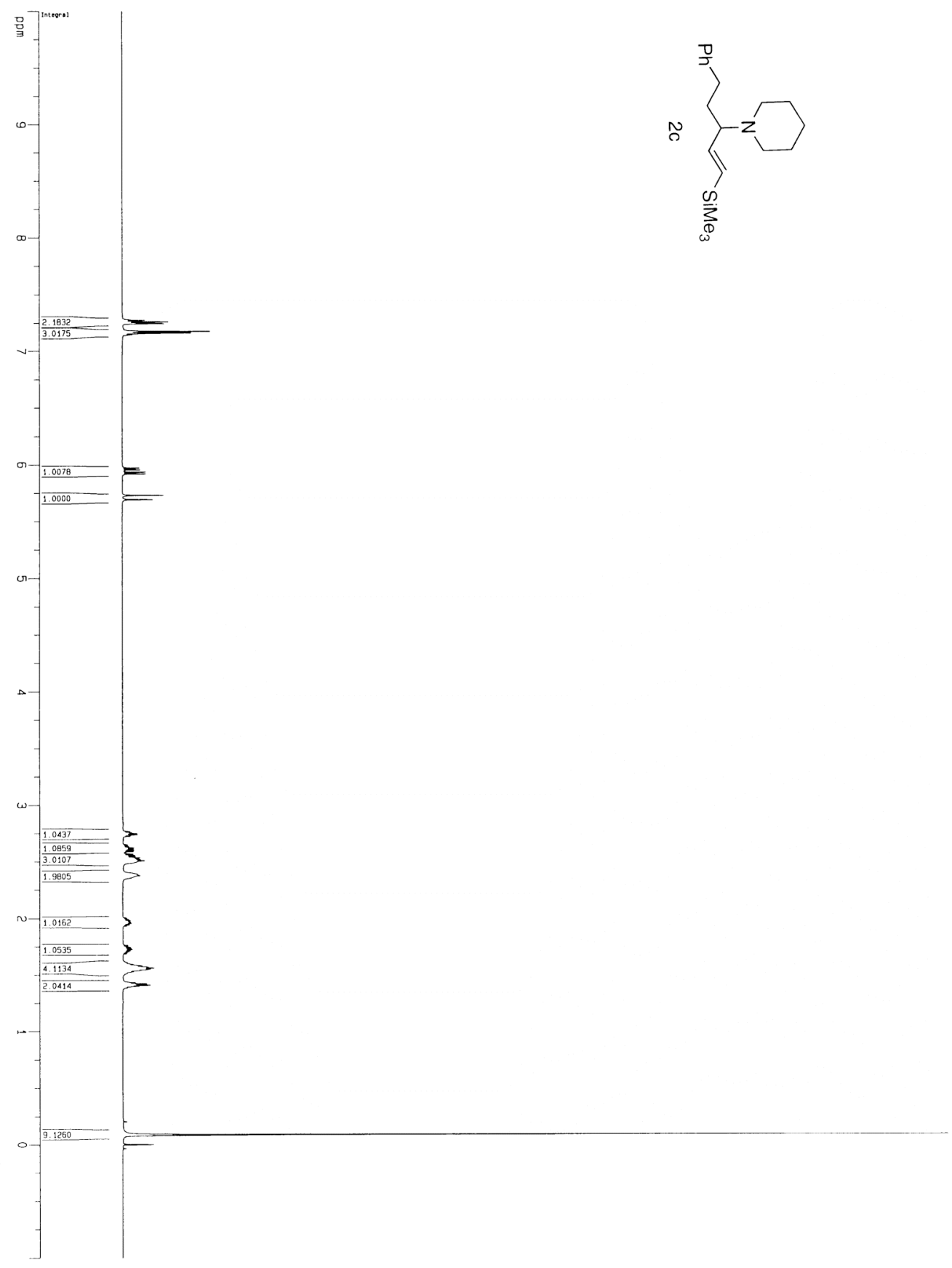




$$
E^{\prime}
$$




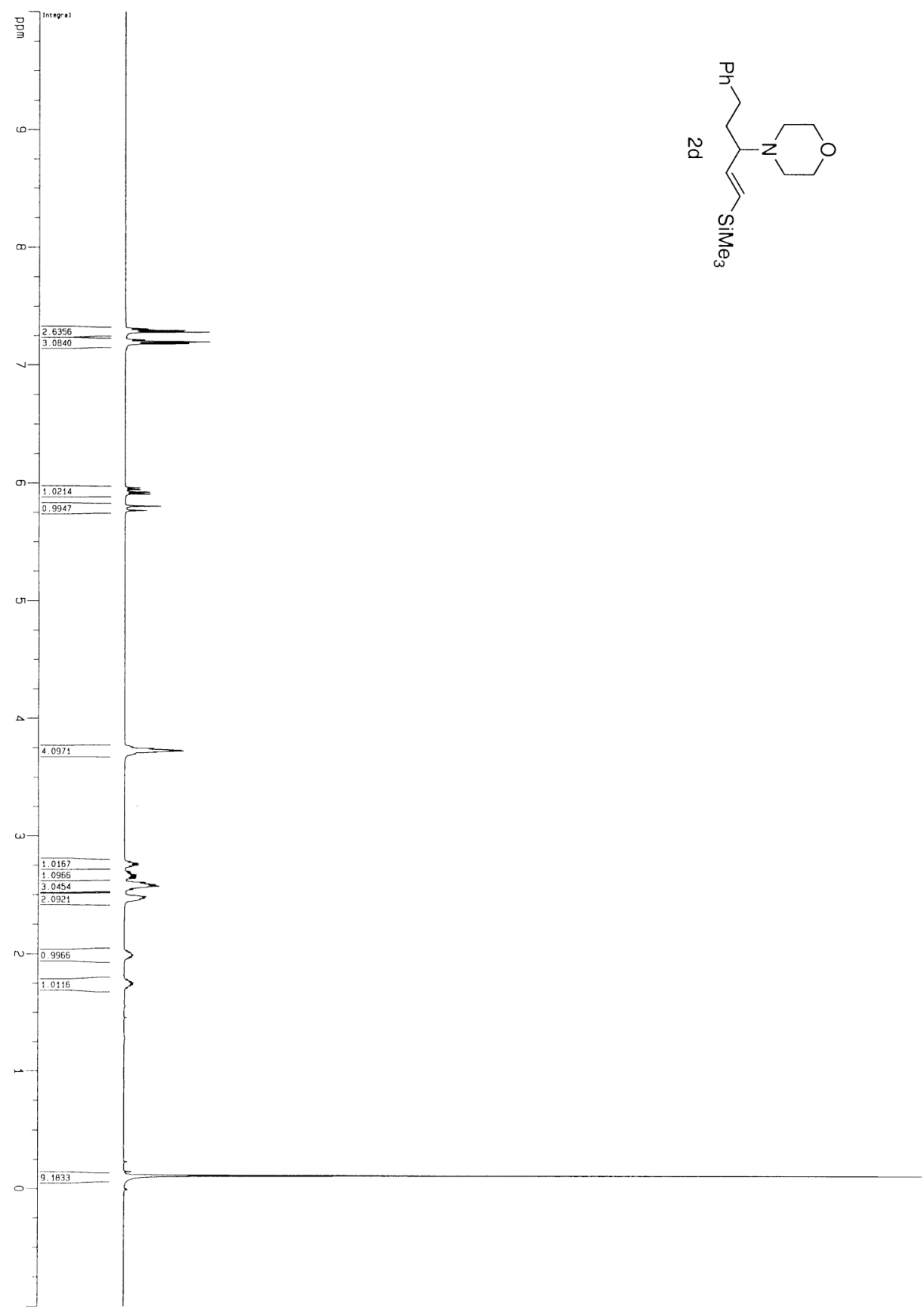




$$
H
$$




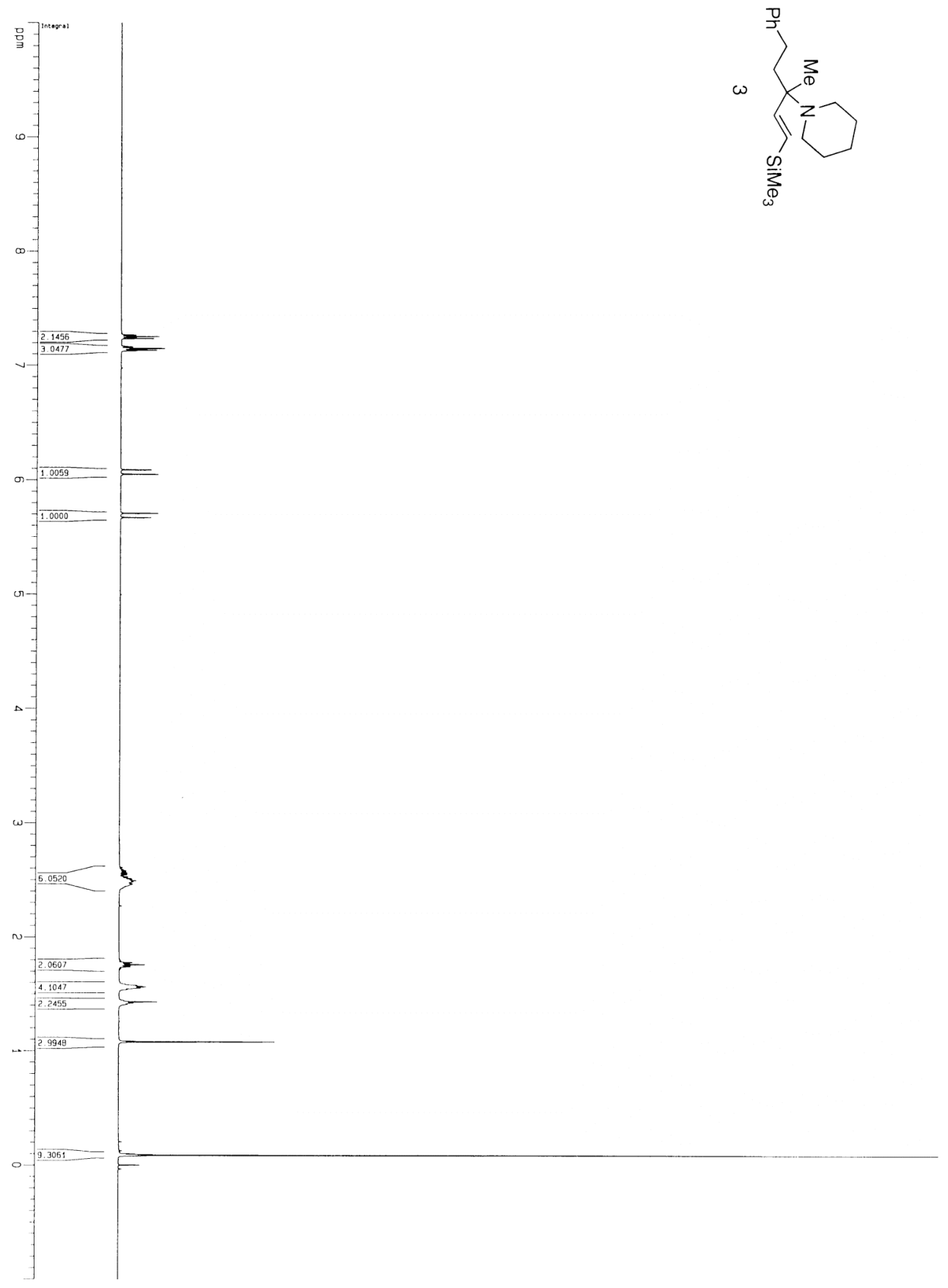




$$
E
$$




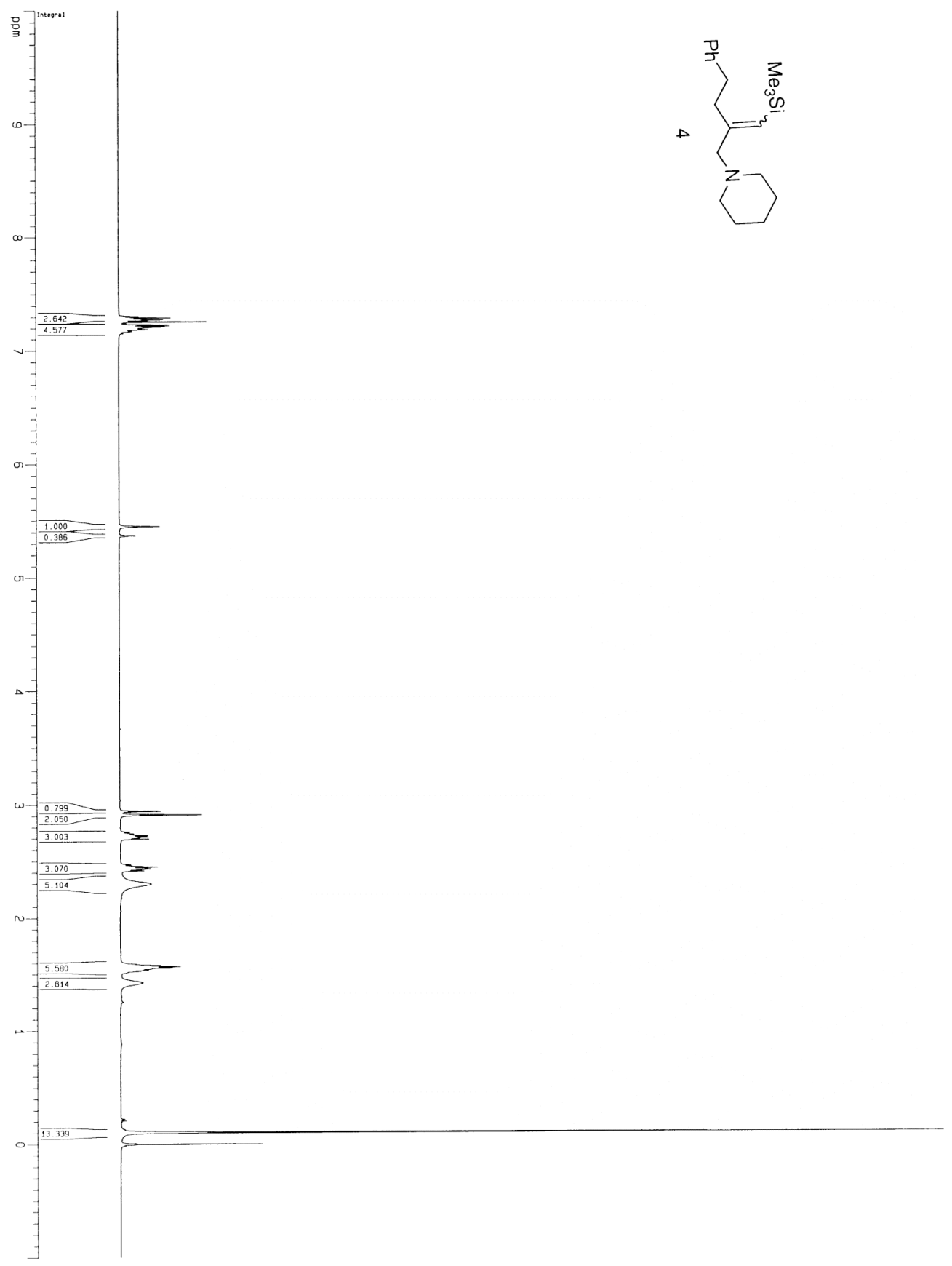




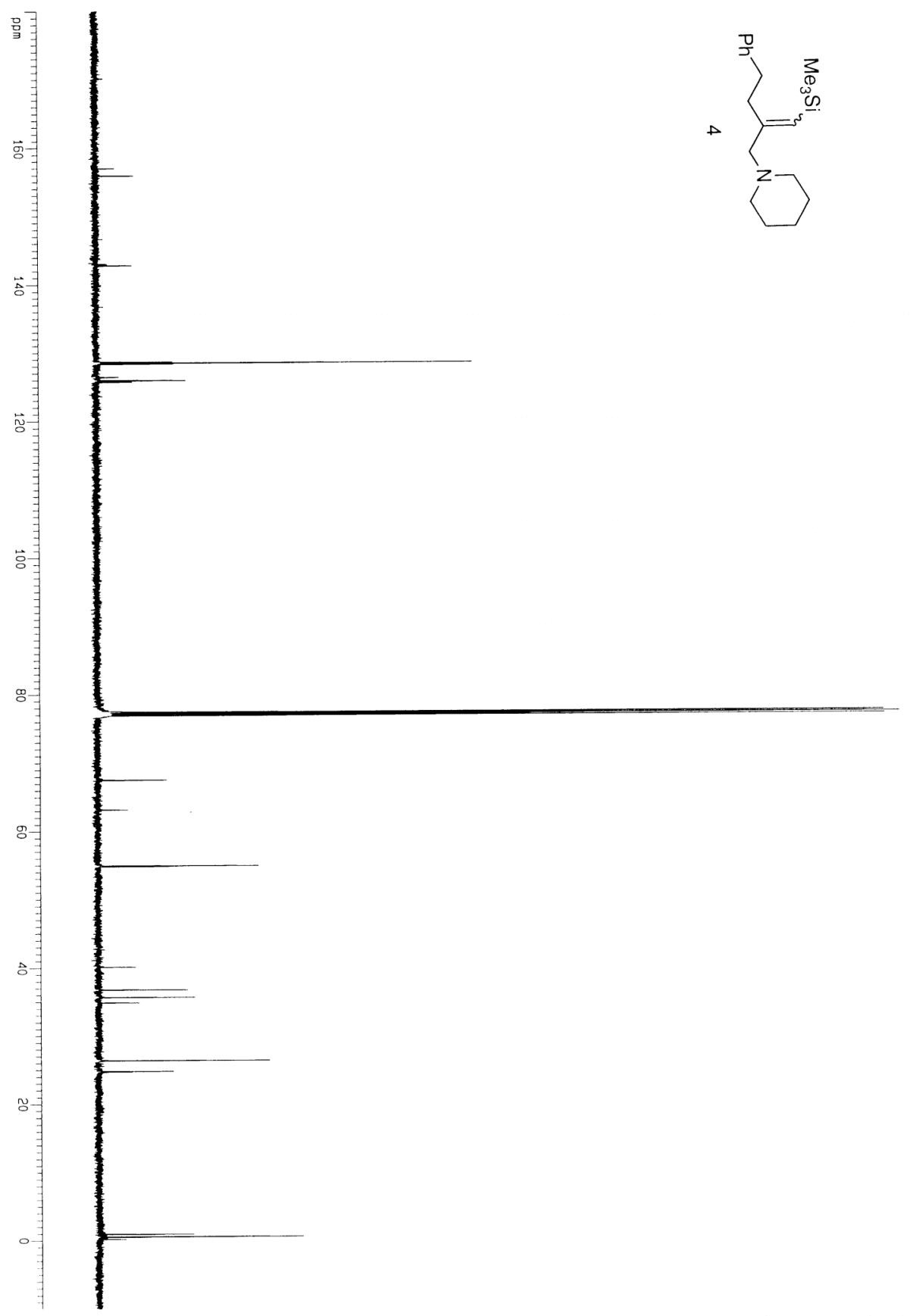




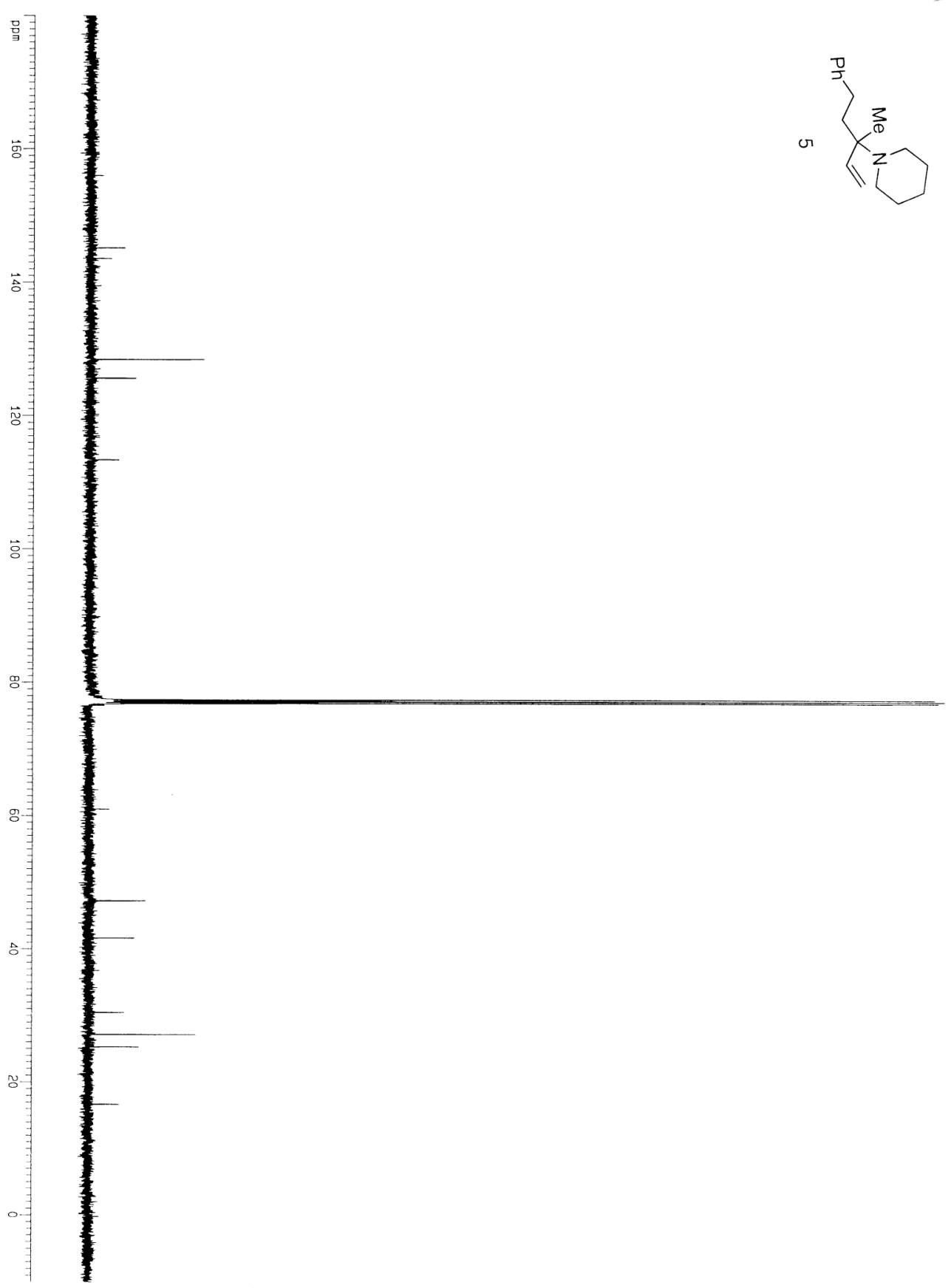




$$
1
$$




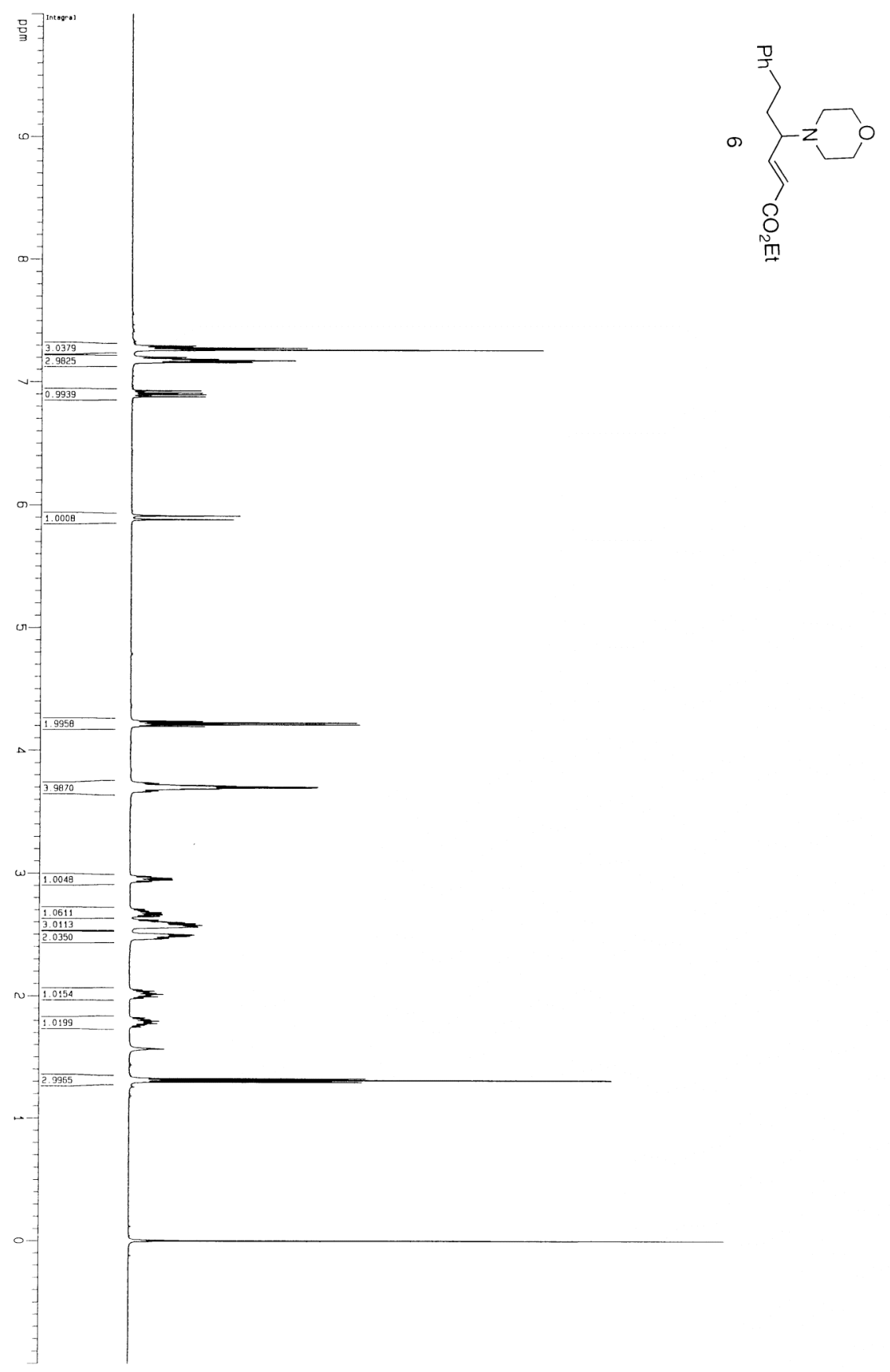




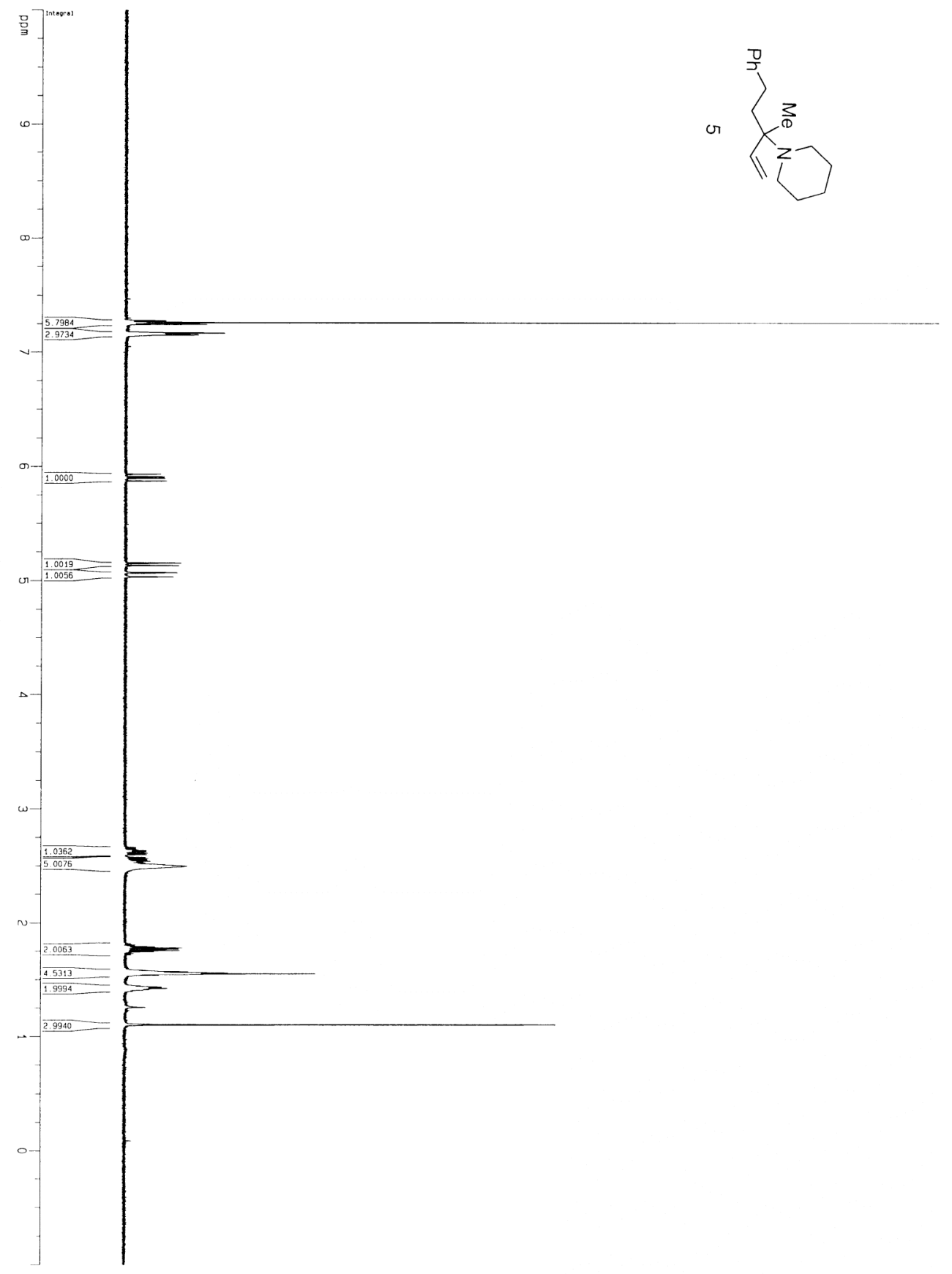

